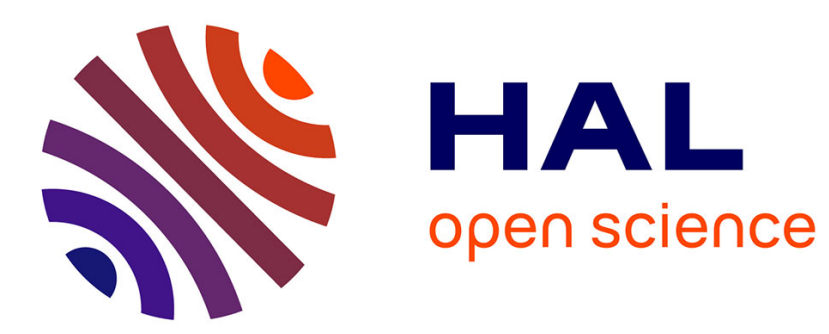

\title{
Fiber-based device for the detection of low-intensity fluctuations of ultrashort pulses
}

\author{
Charles-Henri Hage, Bertrand Kibler, Christophe Finot
}

\section{To cite this version:}

Charles-Henri Hage, Bertrand Kibler, Christophe Finot. Fiber-based device for the detection of low-intensity fluctuations of ultrashort pulses. Applied optics, 2012, 51 (7), pp.949-953. 10.1364/AO.51.000949 . hal-00644853

\section{HAL Id: hal-00644853 \\ https://hal.science/hal-00644853}

Submitted on 25 Nov 2011

HAL is a multi-disciplinary open access archive for the deposit and dissemination of scientific research documents, whether they are published or not. The documents may come from teaching and research institutions in France or abroad, or from public or private research centers.
L'archive ouverte pluridisciplinaire HAL, est destinée au dépôt et à la diffusion de documents scientifiques de niveau recherche, publiés ou non, émanant des établissements d'enseignement et de recherche français ou étrangers, des laboratoires publics ou privés. 


\title{
Fiber-based device for the detection
}

\section{of low intensity fluctuations of ultrashort pulses}

\author{
Charles-Henri Hage, Bertrand Kibler, and Christophe Finot* \\ Laboratoire Interdisciplinaire Carnot de Bourgogne, UMR 5209 CNRS - Université de \\ Bourgogne, Dijon, France \\ *Corresponding author: christophe.finot@u-bourgogne.fr
}

We describe a fiber-based device that can significantly enhance the low intensity fluctuations of an ultrashort pulse train to detect them more easily than with usual direct detection systems. Taking advantage of the Raman intrapulse effect that progressively shifts the central frequency of a femtosecond pulse propagating in an anomalous dispersion fiber, a subsequent spectral filtering can efficiently increase the level of fluctuations by more than one order of magnitude. We show that attention has to be paid to maintain the shape of the statistical distribution unaffected by the nonlinear process.

OCIS codes: $060.4370,060.5530,320.7140$.

\section{Introduction}

Detecting low power fluctuations with an easy-to-implement device is of practical interest for many applications such as optical pulse characterization, jitter measurements or biophotonics. Several solutions have already proved their efficiency such as synchronous detection [1], radio- 
frequency analysis [2] or autocorrelation measurements of the pulse train [3]. However, they require the use of dedicated and onerous instruments. In this context, nonlinear effects acting in a single-mode optical fiber can constitute a simple and efficient alternative. It has been recently proposed to take advantage of the self-phase modulation undergone by picosecond pulses to alloptically magnify the amplitude fluctuations of a pulse train at telecommunication wavelengths [4]. Quite remarkably, it has been shown that the statistical distribution of the amplitude fluctuations is maintained after magnification.

We discuss in this contribution a novel experimental scheme based on the soliton selffrequency shift (SSFS) experienced by a femtosecond pulse evolving into a photonic crystal fiber (PCF). We first present the principle of our method and the associated setup. Next we describe how the statistical distributions are reshaped. Finally we provide experimental results assessing the optical jitter magnification and the ability to increase detection margins.

\section{Principle of operation and experimental setup}

The principle of our jitter magnifier is illustrated in Fig. 1 and relies on the transfer function (TF) that links the incoming power to the output power. In a previous work [4], it has been outlined that if the TF can be locally approximated at a working power $\mathrm{P}_{0}$ by a linear function having a $\mathrm{x}$ intercept $\mathrm{P}_{\mathrm{C}}$, then the relative level of output fluctuations $\Delta \mathrm{P}_{\text {out }} / \mathrm{P}_{\mathrm{C}}$ is linked to the input relative fluctuations $\Delta \mathrm{P}_{\text {in }} / \mathrm{P}_{0}$ by the following relationship :

$$
\frac{\Delta P_{o u t}}{P_{C}}=\frac{\Delta P_{i n}}{P_{0}} \frac{1}{1-P_{C} / P_{0}}
$$

with $\mathrm{P}_{\text {out }}$ being the output power and $\Delta \mathrm{P}_{\text {out }}$ and $\Delta \mathrm{P}_{\text {in }}$ being the output and input levels of fluctuations respectively. From this simple law, amplification of the relative amplitude jitter requires $\mathrm{P}_{C}$ strictly between 0 and $2 \mathrm{P}_{0}$ so that a strictly proportional $\mathrm{TF}\left(\mathrm{P}_{\mathrm{C}}=0\right)$ is prohibited. 
One of the simplest TF shapes that fulfills this requirement is comprised of two linear segments and is illustrated in Fig. 1.

The main experimental issue is to realize a TF having properties close to this ideal shape. The scheme proposed in Ref. [4] has validated the use of the non-proportional TF by demonstrating a 10 times amplitude jitter magnification: the central optical bandpass filtering of a spectrum expanded by self-phase modulation has led to a transfer function that can be locally approximated by a linear increasing segment. We explore here the use of soliton self-frequency shift affecting a femtosecond pulse evolving in a PCF with anomalous dispersion. Thanks to the intrapulse Raman contribution of the silica, the central frequency of the pulse progressively shifts towards longer wavelengths, this frequency shift being basically controlled by the peak power of the initial pulse [5]. Such a spectral shift has been previously used for a wide range of photonics applications such as wavelength-tunable ultrashort sources [6], optical sampling [7], coherent anti-Stokes Raman scattering bioimaging [8] or analog-to-digital converters [9]. Here, we combine the SSFS process with a long-pass filter blocking the low-wavelength components, thus acting as an optical thresholder that efficiently discriminates the pulses according to their initial power. Indeed, the pulses having a peak-power not high enough to be sufficiently frequency shifted are vanished whereas the other pulses pass through the filter. Interestingly, the transition between the two regimes is rapid and close from being linear, which is obviously beneficial for the targeted application.

More precisely, the experimental setup we implemented is depicted in Fig. 2. A femtosecond pulse train is delivered by a passively mode-locked oscillator (Amplitude Systems t-Pulse laser with 48-MHz repetition rate and 190-fs pulse duration) at the initial wavelength of $1033 \mathrm{~nm}$. The PCF input power is controlled with an electro-optic modulator (EOM) driven by 
an electrical arbitrary function generator so that the transmission level of the EOM can be dynamically changed at a repetition rate from several $\mathrm{Hz}$ up to several tens of $\mathrm{kHz}$. The fiber under test is typically a 2-m long solid-core highly nonlinear photonic crystal fiber [10] having a zero-dispersion wavelength well below the pumping wavelength (in our case, we have tested two PCFs, called fibers A and B, with zero-dispersion wavelengths estimated to $710 \mathrm{~nm}$ and $765 \mathrm{~nm}$ respectively). In order to favor the existence of a single soliton, fibers with high dispersion have been chosen $[11,12]$. At the fiber output, a long-pass filtering (with a cutoff wavelength at 1200 $\mathrm{nm}$, transmission function is provided on the inset of Fig. 3) enables to realize the aforementioned thresholding/magnification function. The spectrally filtered signal is then collected by an InGaAs photodiode and monitored by a standard oscilloscope. The electronic bandwidth of both photodiode and oscilloscope is not high enough to resolve the fine temporal details of the femtosecond pulse. The recorded fluctuations correspond to the pulse energy jitter, which can be directly linked to the jitter of the pulse peak power [13]. The output average power is also monitored as well as the optical spectrum (when the optical filter is removed).

\section{Experimental results}

\section{Spectral pulse dynamics}

An experimental example of the spectral evolution of the pulse into the PCF as a function of the initial average power is provided in Fig. 3. As expected, we clearly notice a soliton evolving towards longer wavelengths, the frequency shift being controlled by the power launched into the PCF $[14,15]$. For fiber A and the studied power range, a single frequency-shifted soliton is observed [12]. For an input average power $\mathrm{P}_{0}$ of $40 \mathrm{~mW}$ (injection ratio $\sim 35 \%$ ), half of the soliton has passed through the 1200-nm cutoff wavelength of the filter. 


\section{Thresholding transfer function}

The resulting transfer function is plotted in Fig. 4(a) (solid black line). We can distinguish three different stages of the process that can be easily understood (see also Fig. 3). Indeed, for initial powers below $\mathrm{P}_{1}$, the SSFS is too low so that no part of the soliton passes through the filter. On the contrary, for input powers above $\mathrm{P}_{2}$, the whole soliton passes through the filter and we observe a plateau. Between $\mathrm{P}_{1}$ and $\mathrm{P}_{2}$, the soliton progressively passes through the filter so that the output energy continuously increases and a close to linear behavior is empirically observed. This is of course in this transition region that the optical magnifying will occur. In order to accommodate the highest range of input fluctuations, it is beneficial to work in the middle of this linear segment, i.e. at the working power $\mathrm{P}_{0}$. From the slope at this point, one can anticipate a magnifying factor of 20 as it will be confirmed in next section.

TFs have also been experimentally recorded for cutoff wavelengths of the optical filter ranging from $1050 \mathrm{~nm}$ to $1250 \mathrm{~nm}$. Results are summarized in Fig. 4(a) and clearly confirm that longer the cutoff wavelength of the filter is, higher the threshold is, which is fully consistent with the analysis reported in Fig. 3. We can evaluate the magnification factor undergone by the signal when the working power is chosen in the middle of the linear rising part of the TF (note that the amplitude jitter gain may vary over the TF according to the working power). This magnification factor has been found to be close to constant about $20 \pm 2$ for the different cutoff wavelengths. We can note that for low detunings of the filter (cutoff wavelengths of $1050 \mathrm{~nm}$, grey dotted line) the resulting TF does not present a high linear part, which may be detrimental to maintain the statistical probability distribution of the fluctuations. However, in all cases the largely monotonic behavior of the TF helps to avoid some potential confusions that may arise from the oscillating structure of the typical TF used in [4]. 
The fiber properties can also highly affect the transfer function and the working power needed to obtain the switching. Indeed, fibers with lower dispersion and higher nonlinearity coefficient increase the soliton self-frequency shift and thus lead to a lower thresholding power [12]. Such an effect can be seen on Fig. 4(b) for which we have compared two fibers with the same output filter (cutoff wavelength of $1150 \mathrm{~nm}$ ). The fiber with the lowest dispersion (fiber B, dashed black line) has led to a significant reduction of the input power required to the thresholding. Therefore, input pulses with energies as low as a few hundreds of $\mathrm{pJ}$ (a few $\mathrm{kW}$ of peak-power) can be suitable.

\section{Magnification or reshaping of statistical distributions}

In the rest of this paper, we focus on the configuration based on the fiber A combined with the filter having a cutoff wavelength of $1200 \mathrm{~nm}$ (TF plotted in Fig. 4(a) with solid black line). In order to assess the ability of our device to efficiently magnify the input fluctuations, we applied stochastic fluctuations having Gaussian probability distribution generated by our electric arbitrary generator. The statistical distribution recorded after magnification and the associated electrical eye diagram are plotted on Fig. 5(a) for the magnifier working at power $\mathrm{P}_{0}$. The initial $1.06 \%$ rms fluctuations (corresponding to a distribution with a full width at half maximum of $2.5 \%$ ) are increased at the output to a level of $21 \%$ rms. As assessed by the Gaussian fit (dashed line), the general shape is preserved and a magnifying factor close to 20 is obtained, which is in agreement with the prediction based on the TF. Complementary results have been recorded for another probability distribution, i.e. a uniform distribution with an initial variation of $2.5 \%$ (full width at half maximum). Results recorded after the jitter magnifier (Fig. 5(b)) highlight the preservation of the distribution shape and confirms the amplification factor of 20. 
Additional measurements have pointed out the crucial importance of the working power. Indeed, as highlighted by Fig. 6(a), working with a lower initial power close to the threshold power $\mathrm{P}_{1}$ (see Fig. 4(a)) leads to the generation of heavily tailed statistics that strongly differ from the original Gaussian input stochastic field (a Gaussian fit of the output distribution is not able to reproduce the wings of the distribution). Rare pulses having amplitude more than 60 times higher than the most likely event have therefore been recorded at the output of the device, as also illustrated by the electrical eye-diagram of Fig. 6(a). This confirms the major role that may play the long-pass filter in analyzing statistics as it is done frequently in recent articles dealing with optical rogue waves and optical solitons with extreme red shifts. In particular the results obtained from the rude analysis of the filtered pulse train have to be interpreted with care $[13,16,17]$ (see also Fig. 1 for an illustration of the action of the filter on the statistical distribution when working close to the threshold power). Note that an inverse long tail distribution with a tail towards lower powers can also be generated when working close to the plateau of the device (power $\mathrm{P}_{2}$ ). The physics that is here involved is completely different from the dynamics reported in [18] and can be once again easily understood by considering the action of the TF on the initial probability distribution. We expect that the flexibility brought by our device to provide on demand extreme statistics may be helpful to study the evolution of such extreme statistics in additional nonlinear environment.

\section{Magnification of small fluctuations}

We have finally investigated the ability of the device to efficiently discriminate two levels. For this purpose, we have applied periodic levels with a peak-to-peak fluctuation as small as $1.5 \%$. For such a fluctuation, before the magnifier, the fluctuations are below the noise level of the electronics detection chain (standard photodiode and oscilloscope) and cannot be measured. 
After the magnifier working at power $\mathrm{P}_{0}$, results are plotted on Fig. 7(a) and we can clearly make out the fluctuations that are now spaced by $29.5 \%$, in agreement with the magnifying factor that has been previously demonstrated. Therefore, the optical level difference can now be detected in a straightforward way. We also changed the relative weight of the different levels, as shown on Fig. 7(b) and the recorded results reproduce the relative weight of the pulses. Note that the fluctuations observed on each level are mainly attributed to the stability of the pulse train delivered by the source.

\section{Conclusion}

We have proposed and experimentally demonstrated the principle of a new optical amplitude jitter magnifier based on the power dependence of the Raman soliton self-frequency shift. A typical transfer function exhibiting a marked thresholding behavior and a linear transition between two states is demonstrated. Moreover, thanks to the quasi-linear transition both high magnifying factor and shape-preserving statistical distribution amplification are achieved. Magnification by a factor above 20 of the fluctuations has been demonstrated based on the TF, which is a twofold improvement compared to the previous method described in Ref. [4]. Additional measurements have confirmed the increase by more than one order of magnitude of the fluctuations. The choice of the working power is crucial and for input powers which do not lie in the linear part of the TF, a strong reshaping of the statistical distribution can be observed, leading to heavily tailed statistics.

Our results have been demonstrated at $1030 \mathrm{~nm}$ for input pulse energies below $1 \mathrm{~nJ}$ and were based on silica fibers, but the same technique can be applied in the infrared by pumping a soft-glass fiber in the anomalous dispersion regime [19]. Moreover, we can expect that the 
progress in highly nonlinear waveguides may enable this function to be embedded on a photonic chip in a close future [20] and to decrease the input pulse energies.

\section{Acknowledgements}

We thank the support of the Agence Nationale de la Recherche (ANR SOFICARS and MANUREVA, grants ANR-07-RIB-013-03 and ANR-08-SYSC-019). C-H. Hage acknowledges the support of the Conseil Regional de Bourgogne through his grant "jeune chercheur entrepreneur" scheme. B. Kibler also thanks the support of the Conseil Régional de Bourgogne through the Photcom PARI grant. We thank Hervé Rigneault, Esben Andresen, Kamal Hammani, Julien Fatome and Guy Millot for stimulating discussions.

\section{References}

1. R. Burdett, "Amplitude Modulated Signals - The Lock-in Amplifier", Handbook of Measuring System Design (Wiley, 2005).

2. D. Von der Linde, "Characterization of the noise in continuously operating mode-locked lasers," Appl. Phys. B 39, 201-217 (1986).

3. J. Fatome, J. Garnier, S. Pitois, M. Petit, G. Millot, M. Gay, B. Clouet, L. Bramerie, and J. C. Simon, "All-optical measurements of background, amplitude, and timing jitters for high speed pulse trains or PRBS sequences using autocorrelation function," Opt. Fiber. Technol. 14, 84-91 (2007).

4. C. Finot, and J. Fatome, "All-optical fiber-based ultrafast amplitude jitter magnifier," Opt. Express 18, 18697-18702 (2010). 
5. F. M. Mitschke, and L. F. Mollenauer, "Discovery of the soliton self-frequency shift," Opt. Lett. 11, 659-661 (1986).

6. M. C. Chan, S. H. Chia, T. M. Liu, T. H. Tsai, M. C. Ho, A. A. Ivanov, A. M. Zheltikov, J. Y. Liu, H. L. Liu, and C. K. Sun, "1.2- to 2.2-um Tunable Raman Soliton Source Based on a Cr-Forsterite Laser and a Photonic-Crystal Fiber," IEEE Photon. Technol. Lett. 20, 900-902 (2008).

7. N. Yamada, S. Nogiwa, and H. Ohta, "640-Gb/s OTDM signal measurement with highresolution optical sampling system using wavelength-tunable soliton pulses," IEEE Photon. Technol. Lett. 16, 1125-1127 (2004).

8. E. R. Andresen, V. Birkedal, J. Thogersen, and S. R. Keiding, "Tunable light source for coherent anti-Stockes Raman scattering microspectroscopy based on the soliton selffrequency shift," Opt. Lett. 31, 1328-1330 (2006).

9. C. Xu, and X. Liu, "Photonic analog-to-digital converter using self-frequency shift and interleaving spectral filters," Opt. Lett. 28, 986-988 (2003).

10. P. S. J. Russell, "Photonic-Crystal Fibers," J. Lightw. Technol. 24, 4729-4749 (2006).

11. R. Pant, A. C. Judge, E. C. Magi, B. T. Kuhlmey, M. de Sterke, and B. J. Eggleton, "Characterization and optimization of photonic crystal fibers for enhanced soliton selffrequency shift," J. Opt. Soc. Am. B 27, 1894-1901 (2010).

12. C.H. Hage, B. Kibler, E. R. Andresen, S. Michel, H. Rigneault, A. Courjaud, E. Mottay, J. M. Dudley, G. Millot, and C. Finot, "Optimization and characterization of a femtosecond tunable light source based on the soliton self-frequency shift in photonic crystal fiber." Proc. SPIE 8071 (2011). 
13. C. Lafargue, J. Bolger, G. Genty, F. Dias, J. M. Dudley, and B. J. Eggleton, "Direct detection of optical rogue wave energy statistics in supercontinuum generation," Electron. Lett. 45, 217-219 (2008).

14. D. T. Reid, I. G. Cormack, W. J. Wadsworth, J. C. Knight, and P. S. J. Russell, "Soliton selffrequency shift effects in photonic crystal fibre," J. Mod. Opt. 49, 757-767 (2002).

15. F. Druon, N. Sanner, G. Lucas-Leclin, P. Georges, K. P. Hansen, and A. Petersson, "Selfcompression and Raman soliton generation in a photonic crystal fiber of 100-fs pulses produced by a diode-pumped Yb-doped oscillator.," Applied Optics 42, 6768-6770 (2003).

16. D. R. Solli, C. Ropers, P. Koonath, and B. Jalali, "Optical rogue waves," Nature 450, 1054 (2007).

17. M. Erkintalo, G. Genty, and J. M. Dudley, "On the statistical interpretation of optical rogue waves," Eur. Phys. J. Special Topics 185, 135-144 (2010).

18. D. R. Solli, C. Ropers, and B. Jalali, "Rare frustration of optical supercontinuum generation," Appl. Phys. Lett. 96, 151108 (2010).

19. J. Hu, C. R. Menyuk, L. B. Shaw, J. S. Sanghera, and I. D. Aggarwal, "Maximizing the bandwidth of supercontinuum generation in As2Se3 chalcogenide fibers," Opt. Express 18, 6722-6739 (2010).

20. A. C. Judge, S. A. Dekker, R. Pant, C. Martijn de Sterke, and B. J. Eggleton, "Soliton selffrequency shift performance in As2S3 waveguides," Opt. Express 18, 14960-14968 (2010). 


\section{List of figure captions}

Fig. 1. Principle of the optical jitter magnifier.

Fig. 2. Experimental setup.

Fig. 3. Evolution of the output optical spectrum according to the input average power. White ticks on the $\mathrm{x}$-axis indicate the positions of the filters. The transmission function of the long-pass filter with a cutoff wavelength of $1200 \mathrm{~nm}$ is plotted in the top inset.

Fig. 4. Experimental transfer functions: output average power according to the input average power. (a) TF recorded for PCF A and for various cut-off wavelengths of the output long-pass filter. (b) TF obtained for PCFs A and B, dashed and solid lines respectively.

Fig. 5. Statistical distribution of the fluctuations (probability and amplitude are normalized to 1 for the most likely event) and electrical eye-diagram of the pulse train when working at power $\mathrm{P}_{0}$. Results obtained for a Gaussian initial distribution of the fluctuations (subfigure (a)) are compared with the results based on a uniform distribution (subfigure (b)). The experimental statistical distributions are compared to Gaussian and rectangular fits respectively (dashed lines). The negative oscillation on the right side of the eye-diagrams is due to the electronics of the photodiode.

Fig. 6. Statistical distribution of the fluctuations (probability and amplitude are normalized to 1 for the most likely event) and electrical eye-diagram of the output pulse train. Results obtained for the working power $\mathrm{P}_{1}$ (subfigure (a)) are compared with the results obtained for the working 
power $\mathrm{P}_{2}$ (subfigure (b)). The experimental statistical distributions are compared to Gaussian fits (dashed lines) and the results are plotted with a logarithmic scale for the x-axis. The rms amplitude jitter of the initial pulse train is $10.5 \%$ for subplots (a) and (b).

Fig. 7. Statistical distribution of the fluctuations (left, plotted on a linear scale) recorded at the output of the magnifying device, as well as corresponding electrical eye-diagrams (right). Probability and amplitude are normalized to 1 for the most likely event. The initial periodic sequence of amplitude modulation of the peak powers of the pulses is plotted in inset. 
Fig. 1. Principle of the optical jitter magnifier.

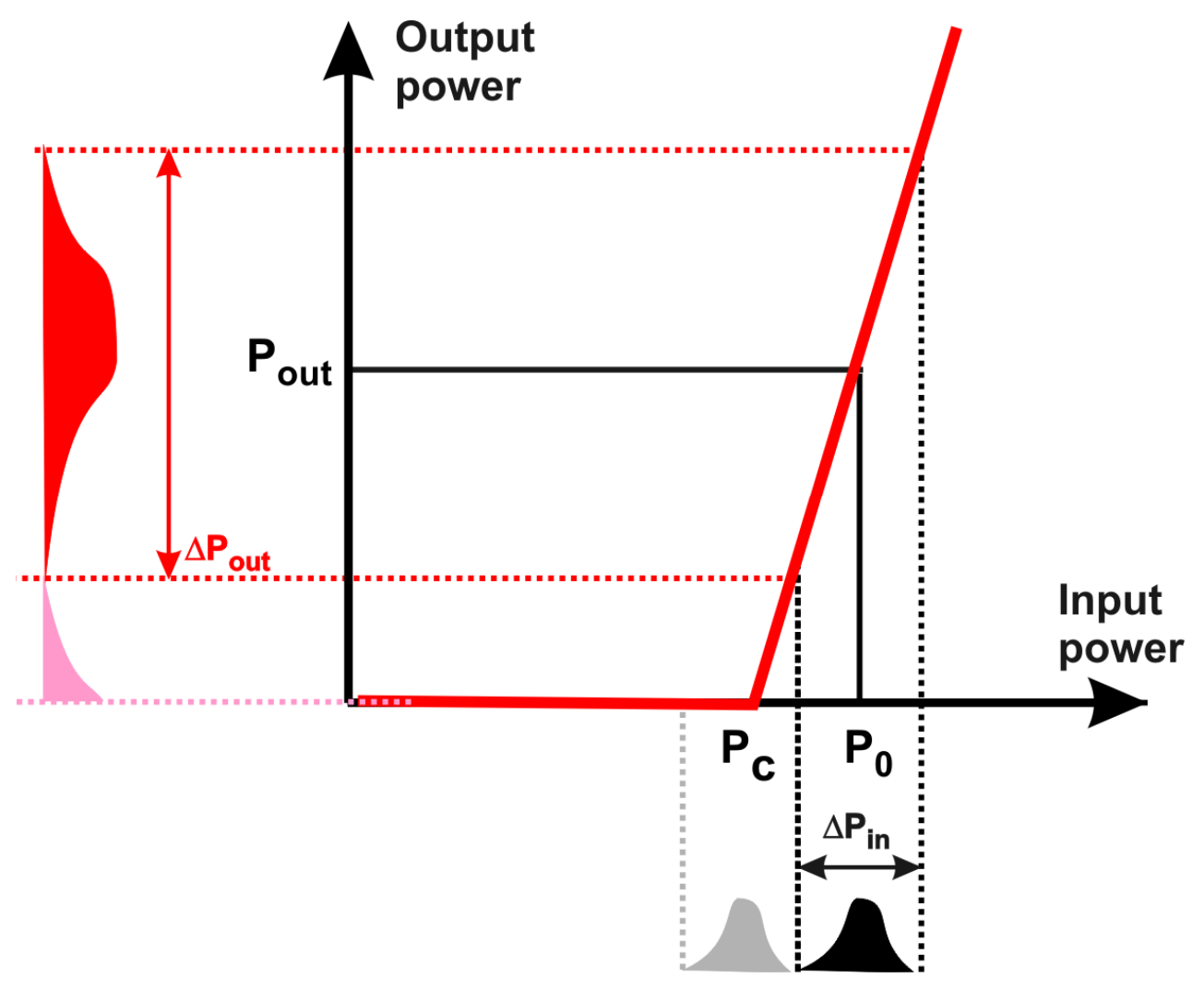


Fig. 2. Experimental setup.

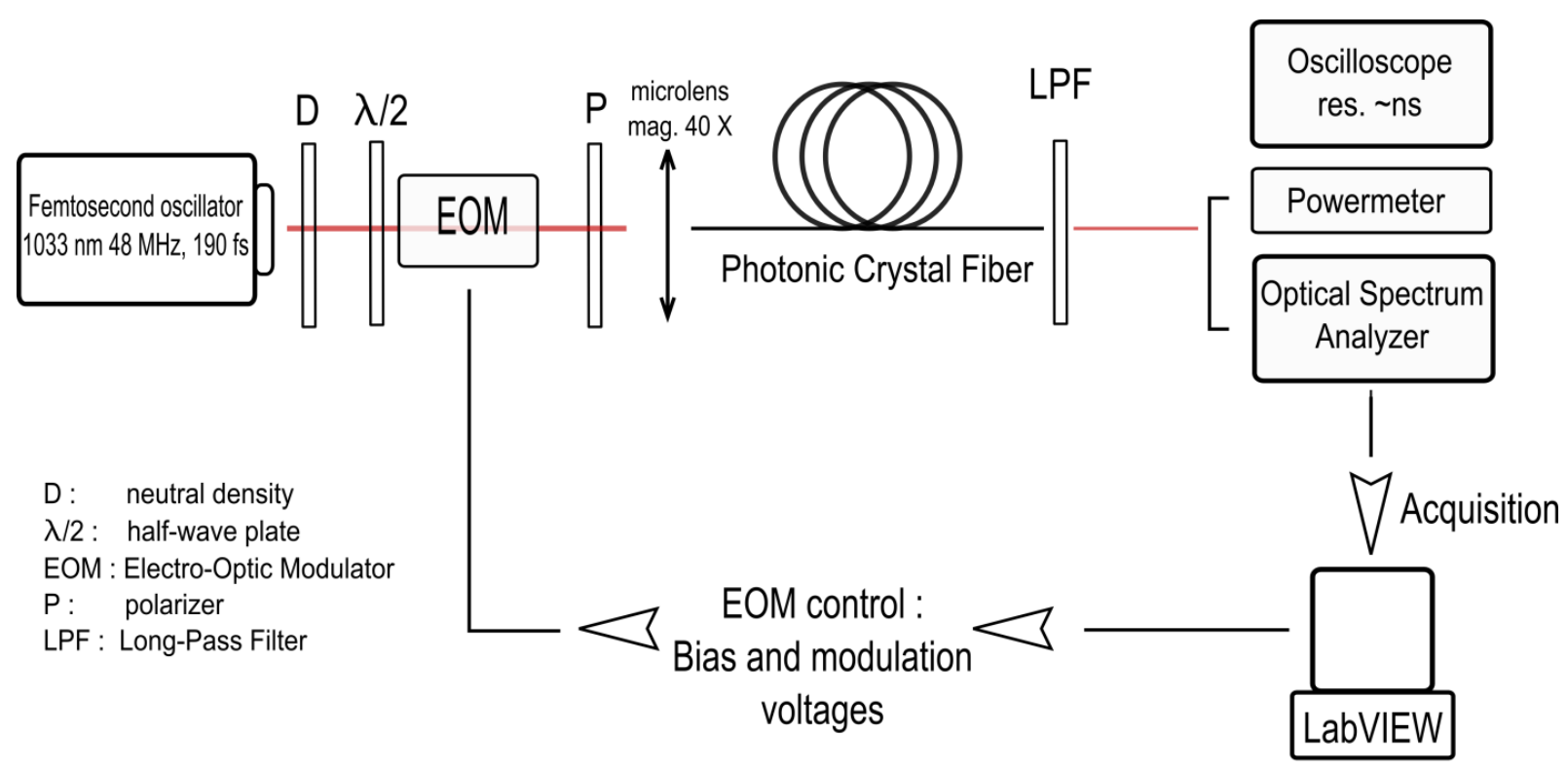


Fig. 3. Evolution of the output optical spectrum according to the input average power. White ticks on the $\mathrm{x}$-axis indicate the positions of the filters. The transmission function of the long-pass filter with a cutoff wavelength of $1200 \mathrm{~nm}$ is plotted in the top inset.

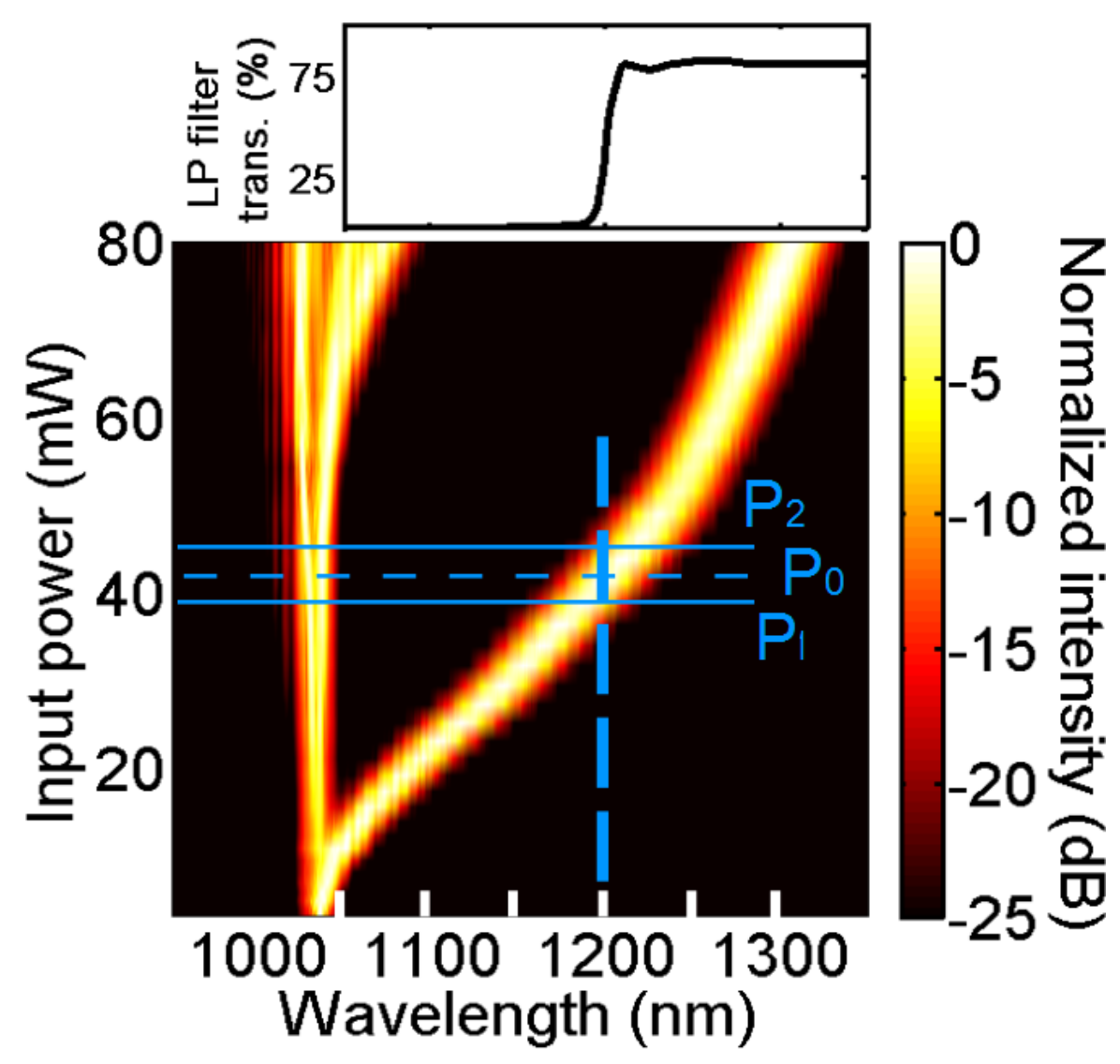


Fig. 4. Experimental transfer functions: output average power according to the input average power. (a) TF recorded for PCF A and for various cut-off wavelengths of the output long-pass filter. (b) TF obtained for PCFs A and B, dashed and solid lines respectively.
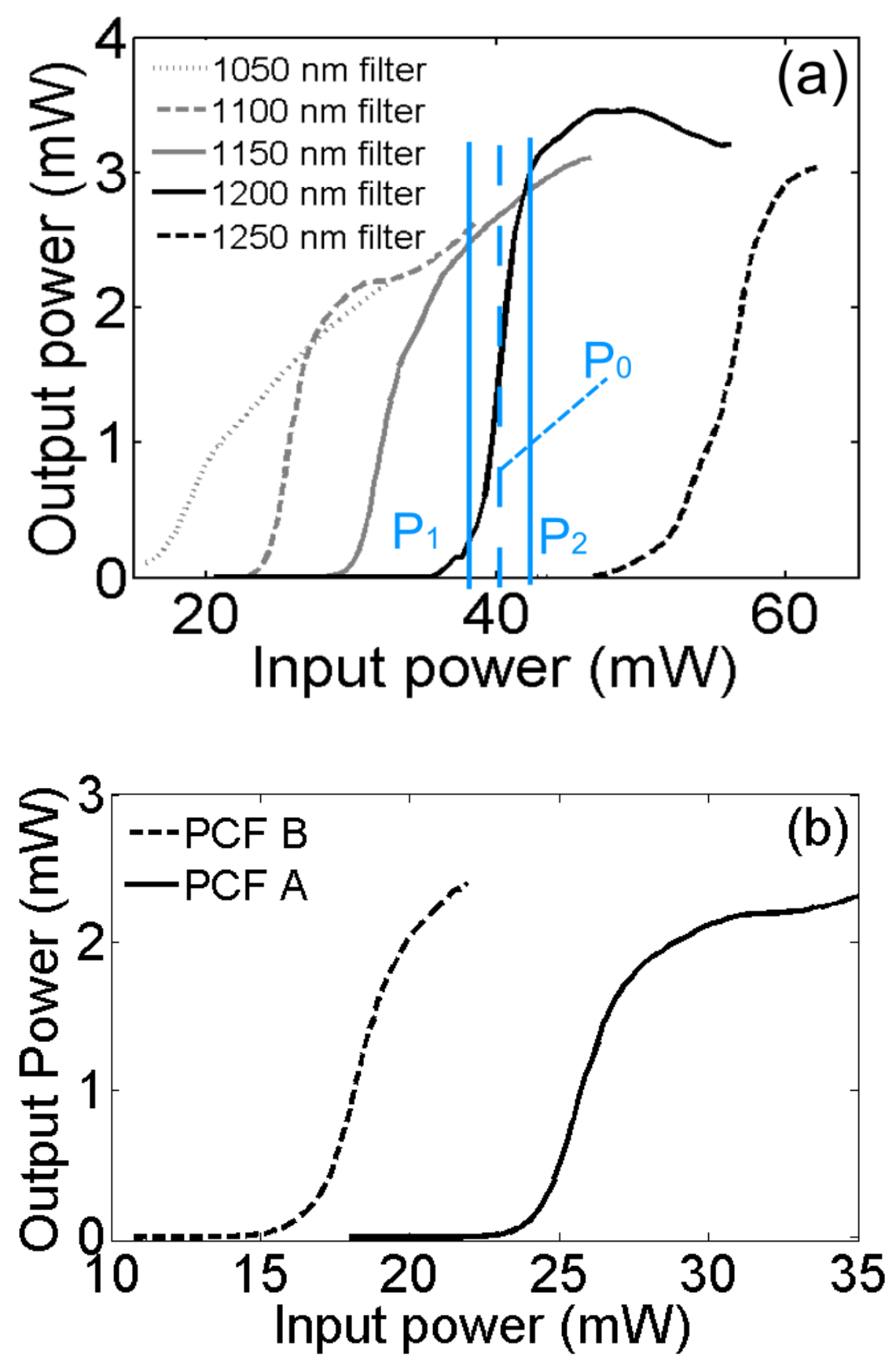
Fig. 5. Statistical distribution of the fluctuations (probability and amplitude are normalized to 1 for the most likely event) and electrical eye diagram of the pulse train when working at power $\mathrm{P}_{0}$. Results obtained for a Gaussian initial distribution of the fluctuations (subfigure (a)) are compared with the results based on a uniform distribution (subfigure (b)). The experimental statistical distributions are compared to Gaussian and rectangular fits respectively (dashed lines). The negative oscillation on the right side of the eye-diagrams is due to the electronics of the photodiode.

(a)
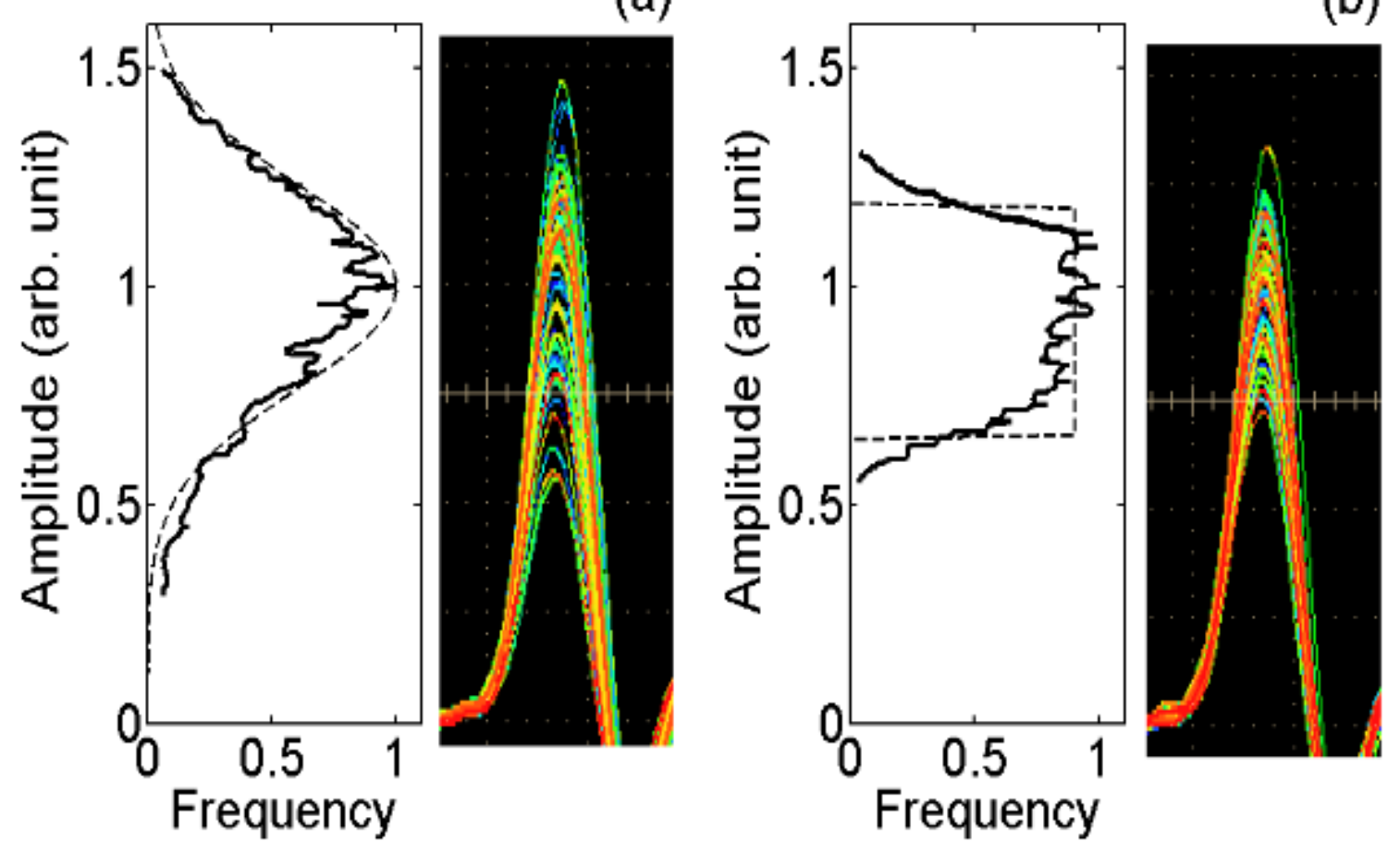
Fig. 6. Statistical distribution of the fluctuations (probability and amplitude are normalized to 1 for the most likely event) and electrical eye diagram of the output pulse train. Results obtained for the working power $\mathrm{P}_{1}$ (subfigure (a)) are compared with the results obtained for the working power $\mathrm{P}_{2}$ (subfigure (b)). The experimental statistical distributions are compared to Gaussian fits (dashed lines) and the results are plotted with a logarithmic scale for the $\mathrm{x}$-axis. The rms amplitude jitter of the initial pulse train is $10.5 \%$ for subplots (a) and (b).
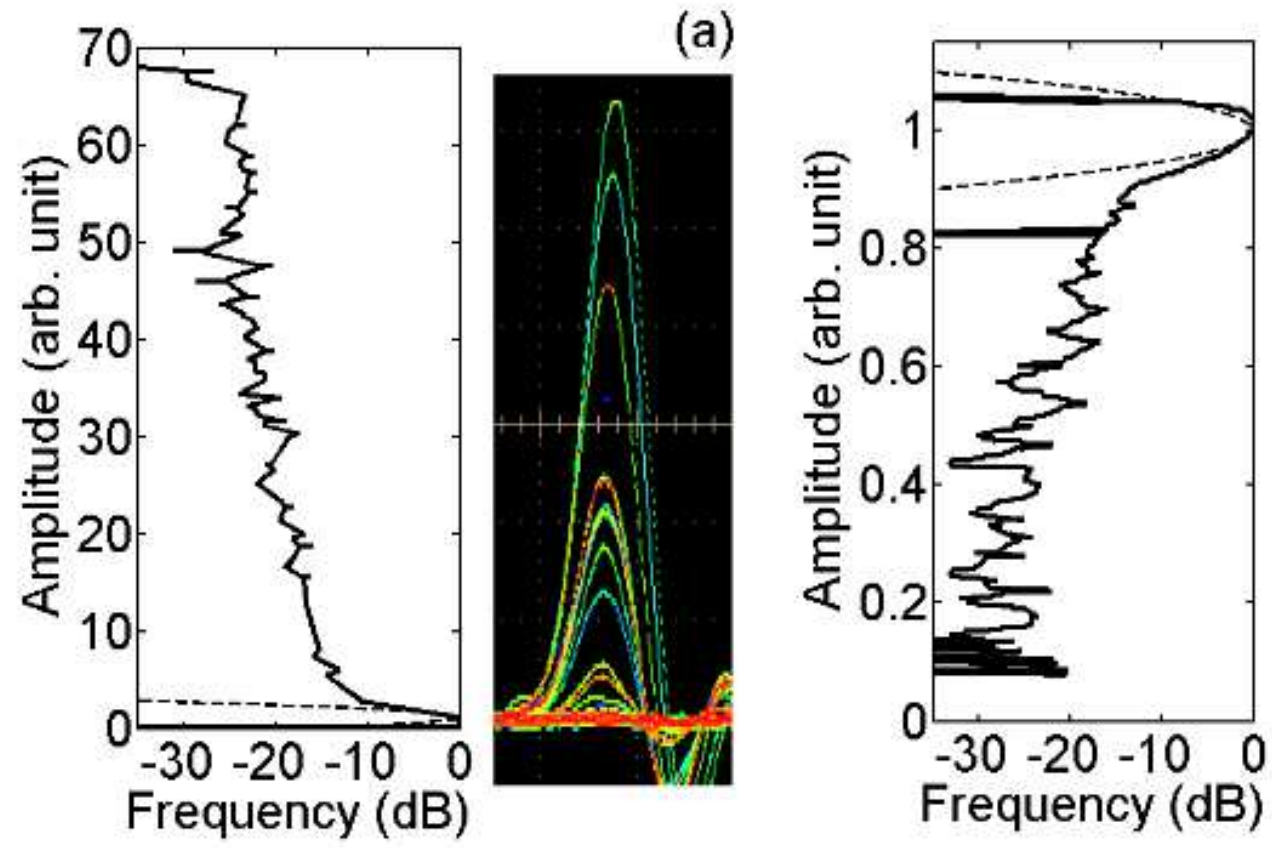

(b)

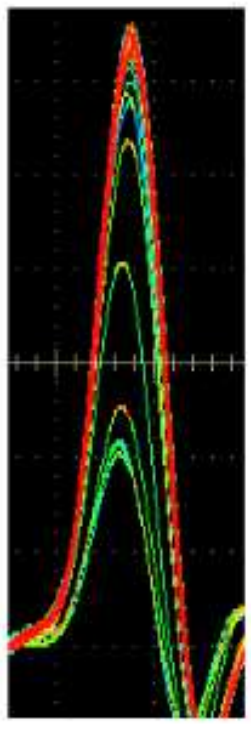


Fig. 7. Statistical distribution of the fluctuations (left, plotted on a linear scale) recorded at the output of the magnifying device, as well as corresponding electrical eye-diagrams (right). Probability and amplitude are normalized to 1 for the most likely event. The initial periodic sequence of amplitude modulation of the peak powers of the pulses is plotted in inset.
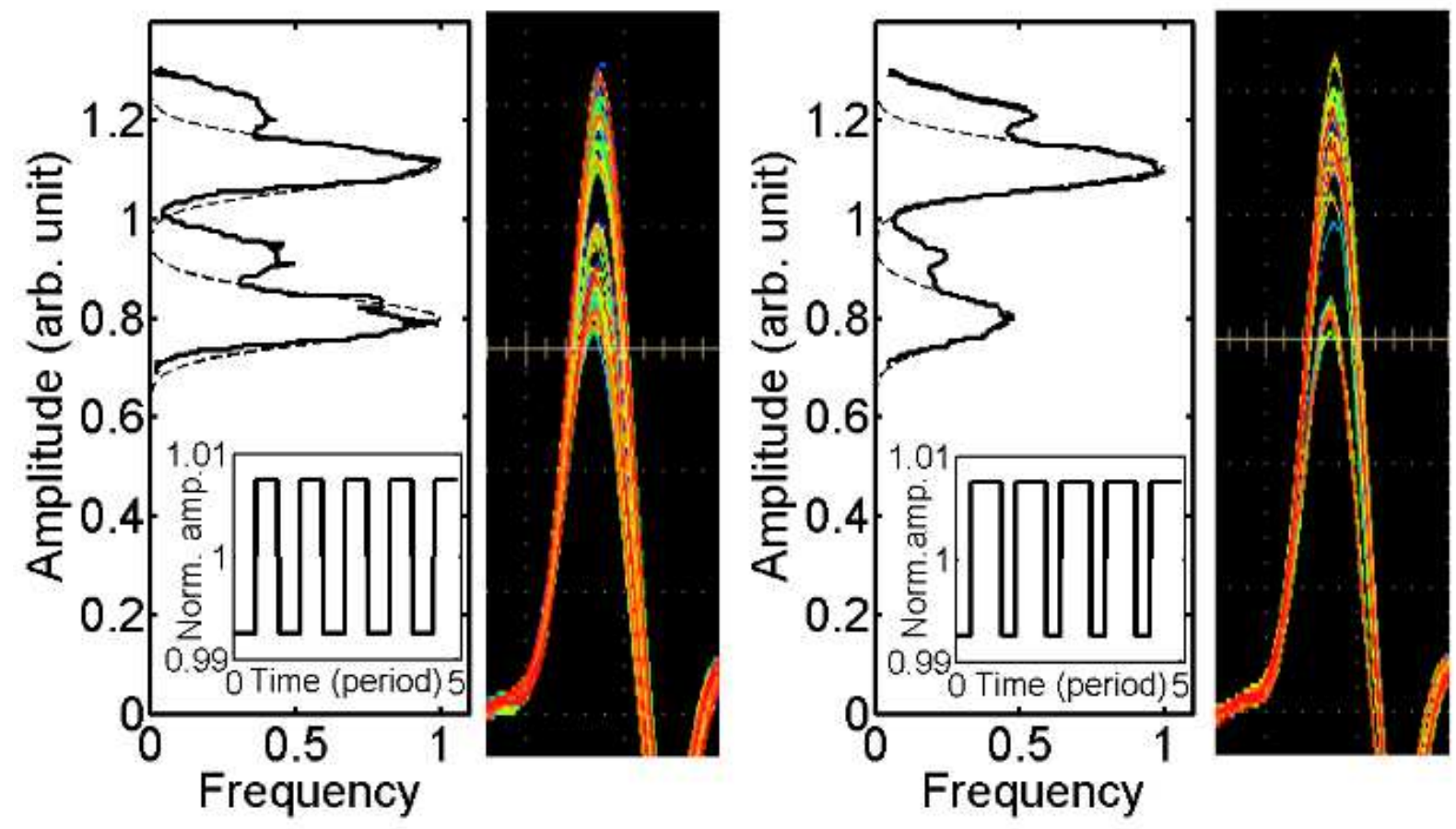